\title{
Association and not semantic relationships elicit the N400 effect: Electrophysiological evidence from an explicit language comprehension task
}

\author{
SINÉAD M. RHODES and DAVID I. DONALDSON \\ The Psychological Imaging Laboratory, Department of Psychology, University of Stirling, Stirling, UK
}

\begin{abstract}
Language comprehension studies have identified the N400, an event-related potential (ERP) correlate of the processing of meaning, modulation of which is typically assumed to reflect the activation of semantic information. However, N400 studies of conscious language processing have not clearly distinguished between meaning derived from a semantic relationship and meaning extracted through association. We independently manipulated the presence of associative and semantic relationships while examining the N400 effect. Participants were asked to read and remember visually presented word pairs that shared an association (traffic-jam), an association+semantic relationship (lemon-orange), a semantic relationship alone (cereal-bread), or were unrelated (beard-tower). Modulation of the N400 (relative to unrelated word pairs) was observed for association and association + semantic word pairs but not for those that only shared a semantic relationship.
\end{abstract}

Descriptors: Association, Event-related potentials, Language, N400, Semantic memory

Theories of language comprehension typically assume that the processing of meaning is based on the "semantic knowledge" that we have about the world. Words are considered to share semantic properties, for example, when they refer to items that have "features" in common (often based on category membership) or shared functional relationships (e.g., cereal-bread or broom-floor). Neuropsychological studies in particular provide compelling evidence that semantic knowledge is organized categorically and functionally (e.g., see Capitani, Laiacona, Mahon, \& Caramazza, 2003) and that these are important elements of semantic knowledge. Consequently semantic knowledge, in the form of categorical and functional features, has formed the basis of theories of language comprehension. Semantic knowledge is thought to drive the processing of meaning in language.

Although semantic knowledge is clearly important for the processing of meaning, close examination of language comprehension studies (e.g., see Federmeier \& Kutas, 1999; Kutas \& Iragui, 1998) suggests that the presence of semantic relationships has often been confounded with associations, where one word calls to mind another based on free association (Postman \& Keppel, 1970). Although associative and semantic relationships often co-occur (e.g., lemon-orange), this need not always be the case; words sharing a semantic relationship need not necessarily

This research was supported by a BBSRC research grant to D.I.D.

Address reprint requests to: Dr. Sinéad Rhodes, The Psychological Imaging Laboratory, Department of Psychology, University of Stirling, Stirling FK9 4LA, UK. E-mail: s.m.rhodes@stir.ac.uk be associated (e.g., cereal-bread), and the associations between words need not necessarily rely on a semantic relationship (e.g., traffic-jam). The distinction between associative and semantic relationships is central to a popular theoretical model of language processing (Collins \& Loftus, 1975). This spreading activation model has suggested that information about associations between words can occur at a lexical level, whereas their meanings and related concepts are represented at a semantic level. In the present study we investigate the distinction between associative and semantic relationships, examining the influence of these two factors on the processing of meaning during an explicit language comprehension task that encourages automatic processing.

Attempts have been made to distinguish between associative and semantic relationships within the behavioral priming literature, where performance reflects nonconscious activation of the underlying representations. Priming studies typically report facilitated recognition for the presentation of a target word (e.g., dog) when it is preceded by a semantically related word (e.g., cat) compared to a neutral or unrelated word (e.g., table; see McRae \& Boisvert, 1998; Moss, Ostrin, Tyler, \& MarslenWilson, 1995; Perea \& Rosa, 2002). Priming effects have been reported for stimuli sharing solely a semantic (McRae \& Boisvert, 1998; Perea \& Rosa, 2002, but see Moss, Ostrin, et al., 1995; Shelton \& Martin, 1992) and associative (Ferrand \& New, 2003; Perea, Gotor, \& Nacher, 1997; Williams, 1996; but see Hodgson, 1991; Thompson-Schill, Kurtz, \& Gabrieli, 1998) relationship. Studies have also reported an "associative boost," with greater priming for words sharing associations and a semantic relationship than for semantic relationships alone (Moss, 
Ostrin, et al., 1995, Shelton \& Martin, 1992). The key point here is that associations and semantic relationships are distinguished within the behavioral priming literature, pointing toward the possibility that this distinction may be important more broadly-including within the context of tasks that require the conscious processing of meaning.

To date it is unclear whether associative and semantic information plays distinct roles during tasks that necessitate the conscious activation of meaning (e.g., during explicit memory tests where participants are encouraged to consciously process the stimuli). As noted above, studies of language comprehension have largely focused on semantic relationships between words. At present it is unknown whether purely associative or semantic relationships are processed differently, or whether there is a boost when both types of relationship are present during conscious processing of meaningful information (equivalent to that seen in unconscious priming studies). The present study was therefore designed to identify whether associative and semantic information differentially contributes to the conscious processing of meaning. Before introducing our specific study in detail, we first describe the event-related potential (ERP) N400 effect, which we use as an online measure of the processing of meaningful information.

Many studies of language comprehension have utilized electrophysiological methods. ERPs (derived from EEG) are an ideal method of studying the processes underlying language comprehension because they provide a real-time record of neural activity, allowing processes to be identified and dissociated on the basis of fine-grained temporal information. ERP studies in healthy participants have identified a neural marker sensitive to the processing of word meaning, known as the N400 effect (e.g., Bentin, Kutas, \& Hillyard, 1995; Federmeier \& Kutas, 1999; Heinze, Muente, \& Kutas, 1998; Kuonios \& Holcomb, 1992; Kutas, 1993; Kutas \& Hillyard, 1980, 1984; Kutas \& Iragui, 1998; Kutas, Lindamood, \& Hillyard, 1984; Luck, Vogel, \& Shapiro, 1996; Neville, Kutas, Chesney, \& Schmidt, 1986). The N400 effect is characterized by a modulation of the N400 component-a negative-going peak in the ERP waveform around $400 \mathrm{~ms}$ poststimulus, maximal over the centro-parietal scalp. To be clear, here we distinguish between the N400 component (the negative-going peak itself) and the N400 effect (a modulation of that component across conditions). Studies employing sentence completion paradigms show that a larger N400 component is elicited if the sentence "The man wore a blue shirt and a yellow ..." finishes with "bucket" rather than "tie", reflecting the mismatch between the word and the previously established context (Kutas \& Hillyard, 1984). Behaviorally this phenomenon is referred to as the "expectancy effect" (Kutas \& Federmeier, 2000).

The N400 effect elicited by the expectancy effect is interpreted by many researchers as reflecting activation of items within a structured semantic network that facilitates understanding of the intended meaning of a sentence. There is evidence, however, that the N400 effect is also produced under automatic processing conditions. For example, the N400 is modulated by words not available for report because they are presented during the attentional blink (Luck et al., 1996) and for unconsciously perceived masked words presented at a very short SOA of $67 \mathrm{~ms}$ (Kiefer, 2002). This has led some researchers (e.g., Kiefer, 2002) to argue that the $\mathrm{N} 400$ can be modulated by automatic processes rather than exclusively reflecting strategic expectancy processes. Thus, in summary, it is clear that the N400 effect has been widely used to provide an index of the processing of meaning during language comprehension.

In studies using either sentences or words as stimuli, it is commonly believed that the N400 effect reflects processing that results from the activation of semantic relationships, be it shared category membership or a functional relationship (e.g., Bentin et al., 1995; Federmeier \& Kutas, 1999; Heinze et al., 1998; Kuonios \& Holcomb, 1992; Kutas, 1993; Kutas \& Hillyard, 1980, 1984; Kutas \& Iragui, 1998; Kutas et al., 1984; Luck et al., 1996; Neville et al., 1986). In many studies, the distinction between associative and semantic relationships is simply ignored; in others the distinction is left ambiguous (e.g., Federmeier \& Kutas, 1999; Kutas \& Iragui, 1998). This assumption is embedded within the literature, however, and has been extended to include a wide variety of task parameters, as is evident in the statement "regardless of the type of response required (including none), ERPs elicited by a category non-member are more negative between 200 and $600 \mathrm{~ms}$ than those elicited by a category member" (Kutas \& Iragui, 1998, p. 457). This statement exemplifies the commonly held assumption that semantic relationships (here specifically of a categorical nature) drive N400 effects. Along similar lines, it has been suggested within sentence contexts that the categorical relationship between the subject and object is a more reliable predictor of the N400 effect than the fit of the item in the sentence context itself (Federmeier \& Kutas, 1999). Clearly, by this view, the presence of a category relationship between words is critical in eliciting modulations of the N400 component. As noted above, however, to date, studies of conscious language comprehension have not distinguished between meaning derived from a semantic relationship and meaning extracted through association. Thus, at present, it is unclear whether a semantic relationship is actually sufficient to produce a modulation of the N400 component at all during conscious processing.

To our knowledge only one study, by Koivisto and Revonsuo (2001), has examined the separate influence of associative and semantic relationships on the N400 effect. This study employed a priming paradigm. Unfortunately this study did not include an associative + semantic condition. Using unrelated prime-targets as a baseline, Koivisto and Revonsuo reported reduced negativity during 375-500 ms for prime-targets related by association, but not for those related by a semantic relationship. Moreover, the associative stimuli used in this experiment were exclusively comprised of transparent compound words (e.g., wind-mill), which arguably are confounded by a semantic relationship because the meaning of the pair is related in an obvious and direct way to the meanings of the individual words. Although Koivisto and Revonsuo's findings suggest a distinction between the unconscious processing of associative and semantic relationships, it remains unclear whether a similar distinction can be drawn during tasks that require conscious processing. In the present study we independently manipulated the presence of associative and semantic relationships between word pairs during an explicit memory learning paradigm that required the conscious processing of meaningful information. Based on the common conceptions of the N400 effect described above, we predicted a clear effect for semantically related pairs and set out to discover whether an equivalent effect would be seen for associatively related pairs. Further, in light of reports of an "associative boost" in the priming literature we predicted a larger N400 effect for associative + semantic word pairs in comparison to semantic word pairs. To our surprise, neither of these predictions turned out to be true. 
Table 1. Examples of Word Pairs for Each Relationship Type

\begin{tabular}{lcll}
\hline \hline Association & Association+Semantic & Semantic & Unrelated \\
\hline traffic-jam & lemon-orange & cereal-bread & beard-tower \\
fountain-pen & stool-chair & violin-guitar & stereo-thumb \\
mars-bar & brother-sister & cow-goat & honey-canvas \\
spark-plug & lion-tiger & prince-duke & priest-essay \\
glow-worm & gold-silver & pig-chicken & alarm-cloud \\
spare-tire & comb-brush & sheep-rabbit & tennis-pot \\
grave-digger & foam-rubber & fork-plate & tractor-drug \\
dolly-bird & needle-thread & broom-floor & planet-jeep \\
\hline \hline
\end{tabular}

\section{Method}

\section{Participants}

Thirty-two right-handed students participated for course credit or payment. Informed consent was collected in line with Stirling University Ethics procedures. A recognition memory test was included in the experiment design to ensure attendance to the word pairs during study. An exclusion criterion of recognition performance during the memory test of 2 standard deviations below the mean led to data from 7 participants being discarded. The criteria were based on average performance of $74 \%$ correct and a standard deviation of $13 \%$ in each condition during the recognition memory test, providing a cut-off of $48 \%$, importantly above chance probability. The mean age of the remaining 25 participants was 21 years (range 18-31), 15 of whom were female. All participants were right-handed and had normal or corrected-to-normal vision.

\begin{abstract}
Materials
Four hundred and eight word pairs were created from nouns, verbs, and adjectives (three to nine letters in length) taken from words listed in the MRC Psycholinguistic database. We compared the ERPs elicited by four types of word pairs, (1) "unrelated" randomly paired words sharing no meaningful relationship (e.g., beard-tower), employed as a common baseline, (2) "semantic" words sharing categorical or functional features independent of association (e.g., cereal-bread), (3) "association" words that are associated but do not share a semantic relationship (e.g., traffic-jam), and (4) "association + semantic" words that are associated and also share a semantic relationship (e.g., lemon-orange; see Table 1 for further examples). Importantly, all conditions were statistically matched for word frequency using the Kucera and Francis (1967) frequency norms: word $1, F(3,303)<1, p>.05$; word $2, F(3,303)=2.5$,
\end{abstract}

$p>.05$, and for imagability using the MRC Psycholinguistic database: word $1, F(3,303)<1, p>.05$; word $2, F(3,303)=1.34$, $p>.05$. All conditions were matched for both the presence and absence of associations and semantic relationships (see Table 2). Word classes (proportion of nouns, verbs, and adjectives) are evenly distributed across conditions (see Table 2) and all conditions were matched on word length: word $1, F(3,303)<1, p>.05$; word $2, F(3,303)=2.5, p>.05$.

Word pairs were formed using online databases that provide an assessment of the semantic and associative properties of the words. The presence of a semantic relationship between members of a word pair is defined by the presence of shared category membership or shared function. The degree of semantic relationship present within each word pair was indexed using a semantic space model, which represents the meaning of words numerically. This index is derived individually for each word by measuring the frequency distributions of other words occurring in the immediate context of that word, computed over a large language corpus (containing millions of words; Huettig, Quinlan, McDonald \& Altmann, 2006; McDonald, 2006). Using this model we matched word pairs in the semantic and associative + semantic conditions to have a high index of semantic relatedness, whereas those in the pure associative and unrelated conditions were equivalently low. Association ratings were taken from the Edinburgh Association Thesarus (EAT; Word Association Thesaurus, n.d.), a published word production norm that gives the proportion of participants who called to mind the second word on presentation of the first (i.e., association rank). The EAT was chosen based on its established use in the literature (e.g., Coulson, Federmeier, Van Petten, \& Kutas, 2005) and because rank of association is regarded as a more optimal measure of association than association frequency (Anaki \& Henik, 2003). As with the semantic distance model, the EAT provides a numerical index that allows conditions to be matched. Word pairs characterized by association and association + semantic relationship had equivalent high levels of association, whereas word pairs characterized purely by a semantic relationship had no associative relationship as indicated by this measure.

\section{Procedure}

The ERP data reported in this article correspond to ERPs collected in study phases of the experiment. Participants were visually presented with word pairs of each of the four relationship types for $1500 \mathrm{~ms}$ and were asked to read and remember them. Word pairs were displayed in white font against a black background, in uppercase 18-point courier new font, one word above

Table 2. Stimulus Construction

\begin{tabular}{|c|c|c|c|c|c|}
\hline & Association & Association and semantic & Semantic & Unrelated & $p$ \\
\hline Semantic distance & $.178(.007)$ & $.513(.01)$ & $.523(.007)$ & $.168(.006)$ & $\mathrm{N} / \mathrm{A}$ \\
\hline Association strength & $.206(.01)$ & $.209(.013)$ & 0 & 0 & $\mathrm{~N} / \mathrm{A}$ \\
\hline \multirow[t]{2}{*}{ Frequency } & Word1: 5.47 (1.67) & Word1: 5.18 (1.39) & Word1: $5.32(1.34)$ & Word 1: $5.56(1.62)$ & \\
\hline & Word2: $4.81(1.41)$ & Word2: $5.0(1.57)$ & Word2: 5.53 (1.69) & Word2: $5.24(1.25)$ & \\
\hline Nouns (\%) & 74.0 & 73.0 & 73.5 & 77.0 & $p>.05$ \\
\hline Verbs $(\%)$ & 9.8 & 13.7 & 11.3 & 8.3 & $p>.05$ \\
\hline Adjectives(\%) & 16.2 & 13.2 & 15.2 & 14.7 & $p>.05$ \\
\hline
\end{tabular}

Semantic distance ratings are a numerical representation of the meanings of words, derived from the frequency distributions of the words occurring in the immediate context of a target word, computed over a large language corpus (containing millions of words; Huettig et al., 2006; McDonald, 2006). Semantic distance ratings range from .01 (low semantic) to 1.0 (high semantic). Association strength refers to the proportion of participants who called to mind the second word as a first response on presentation of the first word. The measure employed to construct associative ratings provides ratings of primary rank of associates (Word Association Thesaurus, n.d.). The data displayed show means and standard deviations. The data also show that word classes are evenly distributed across experimental conditions. 
the other, in central vision, using E-Prime (Psychology Software tools). At the viewing distance of $97 \mathrm{~cm}$, the stimuli subtended a maximum horizontal visual angle of approximately $3.7^{\circ}$ and a maximum vertical visual angle of approximately $1.4^{\circ}$. Each participant completed a practice block, followed by 17 experimental blocks, each of which involved a study and test phase. Each study phase involved the presentation of 16 word pairs with equal proportions of the four word-pair types, presented in random order. Prior to each study phase, participants were instructed to read each word pair and to remember them as a pair. This task was chosen with the intention of ensuring that the words were attended to such that the meaning of the stimuli was processed and that compliance with this requirement could be assessed using a later recognition test. Each trial began with an initial fixation cross (+), displayed in the center of the screen for $1000 \mathrm{~ms}$, maintaining participants' fixation and indicating the presentation of the next word pair. Following a 1000-ms blank screen the word pair was presented for $1500 \mathrm{~ms}$, again followed by a blank screen. Each test block involved the presentation of 24 word pairs, 16 of which had been presented in the previous study phase (of which 8 were the same as presented at study and 8 were in a different pairing [rearranged] from study), and 8 were entirely new. Each test trial began with a 1000 -ms fixation cross, followed by a 1000 -ms blank screen. The word pair was presented for 2000 ms, which was followed by a 1500-ms blank screen. Participants had to make a response of same, rearranged, or new during the presentation interval, providing an assessment of participants' recognition of the word pairs.

\section{ERP Recording Parameters}

Scalp EEG was recorded from 61 sites based on an extension of the international 10-20 system (Jasper, 1958; FZ, FCZ, CZ, CPZ, PZ, POZ, Oz, FP1, FP2, AF7, AF8, AF3, AF4, F7, F8, F5, F6, F3, F4, F1, F2, FT7, FT8, FC5, FC6, FC3, FC4, FC1, FC2, T7, T8, C5, C6, C3, C4, C1, C2, TP7, TP8, CP5, CP6, CP3, CP4, CP1, CP2, P7, P8, P5, P6, P3, P4, P1, P2, PO7, PO8, PO5, $\mathrm{PO} 6, \mathrm{PO} 3, \mathrm{PO} 4, \mathrm{O} 1, \mathrm{O} 2)$ using a left mastoid reference. EEG was also recorded from the right mastoid, allowing algebraic reconstruction to an average mastoid reference. Vertical and horizontal EOG were recorded from bipolar pairs of electrodes placed above and below the left eye and on the outer canthi, allowing blink artifacts to be corrected. Interelectrode impedance levels were below $5 \mathrm{k} \Omega$. Data were filtered $(0.01-40 \mathrm{~Hz}$ bandpass $)$ and digitized (16 bit) at a rate of $8 \mathrm{~ms}$ per point.

\section{Data Analysis}

Eye movements were removed from EEG using a commonly employed method (Scan 4.2, 2001). This method consists of a regression analysis in combination with artifact averaging to produce a reliable and valid method for artifact removal (Semlitsch, Anderer, Schuster, \& Presslich, 1986). Blinks were corrected using a minimum of 32 blinks for each participant. No differences were observed on the HEOG channel across conditions (see Figure 1). Individual 1936-ms epochs were formed (beginning with a 104-ms prestimulus baseline), and epochs with baseline drift exceeding $75 \mu \mathrm{V}$ or whose base-to-peak amplitude exceeded $100 \mu \mathrm{V}$ were rejected. Averaged ERP waveforms were baseline corrected and smoothed over a five-point kernel. The mean number (and range of) of trials contributing to the average ERPs were as follows: association, 52 (35-67); association + semantic, 52 (39-66); semantic, 52 (36-65); and unrelated, 53 (3966). Analysis was performed on mean voltage data relative to the prestimulus baseline period using repeated measures ANOVA, and only significant effects involving the factor of relationship type are reported. The Geisser-Greenhouse correction for nonsphericity of data was applied as appropriate, and corrected $d f$ and $F$ values are reported.

\section{Results}

\section{ERPs}

Average ERP waveforms time-locked to the presentation of each type of word pair at study are shown in Figure 1, demonstrating a clear negative-going peak around $400 \mathrm{~ms}$ poststimulus. The waveforms diverge from about $300 \mathrm{~ms}$ poststimulus onset, with the ERPs for association and association + semantic conditions becoming less negative than the unrelated condition, a difference that is maximal over central sites during the typical N400 time window of 300 to $500 \mathrm{~ms}$ as shown in Figure 2. This pattern of data clearly reveals a modulation of the N400 component for both association conditions. In contrast, there is no divergence in the ERPs for the semantic and unrelated conditions during 300 to $500 \mathrm{~ms}$, as is clear from the overlap between the semantic and unrelated waveforms shown in Figure 2. The N400 effect for association and association + semantic conditions continues after $500 \mathrm{~ms}$, and although the waveforms for semantic and unrelated pairs also begin to diverge at approximately $600 \mathrm{~ms}$, the activity seen for the semantic condition appears to reflect a quite different effect, namely, a shift over posterior parietal electrode sites that can be seen for all three of the relationship types from around 500 to $1100 \mathrm{~ms}$, as shown in Figure 3, here referred to as a left parietal effect. The topography of this left parietal effect is shown for the semantic condition in Figure 4.

\section{ERP Analyses}

300-500 ms. In line with the previous literature, analyses to investigate N400 effects were quantified from 300 to $500 \mathrm{~ms}$ poststimulus. An ANOVA was conducted with the factors of relationship type (association, association+semantic, semantic, unrelated), hemisphere (left, right) laterality (medial: sites 1 and 2; lateral: sites 5 and 6), and location (f, fc, c, cp, p, po lines) as shown in Figure 5. The ANOVA revealed significant Relationship Type $\times$ Hemisphere, $F(3,72)=4.4, p<.007$, Relationship Type $\times$ Laterality, $F(3,72)=4.14, p<.009$, and Relationship Type $\times$ Laterality $\times$ Location, $F(15,360)=1.87, p<.03$, interactions. Given the presence of a significant three-way Relationship Type $\times$ Laterality $\times$ Location interaction, subsidiary ANOVAs were conducted separately on each of the six locations, with conditions of relationship type and laterality (collapsed across hemisphere).

The follow-up analyses revealed that the original significant three-way interaction reflected Relationship Type $\times$ Laterality interactions at the fronto-central, $F(3,72)=5.2, p<.003$, central, $F(3,72)=8.94, p<.001$, and centro-parietal, $F(3,72)=4.04$, $p=.01$, locations. Follow-up ANOVAs revealed that the significant Relationship Type $\times$ Laterality interaction at the central location reflected a significant effect at medial sites alone, $F(2.1,50.3)=3.28, p<.04$. Pairwise comparisons showed this reflected reduced negativity for the association and association + semantic conditions in comparison to the unrelated condition, and, contrary to our predictions, the association and association + semantic conditions also differed from the semantic condition (all $p \mathrm{~s}<.05$ ). Furthermore, there was no significant 


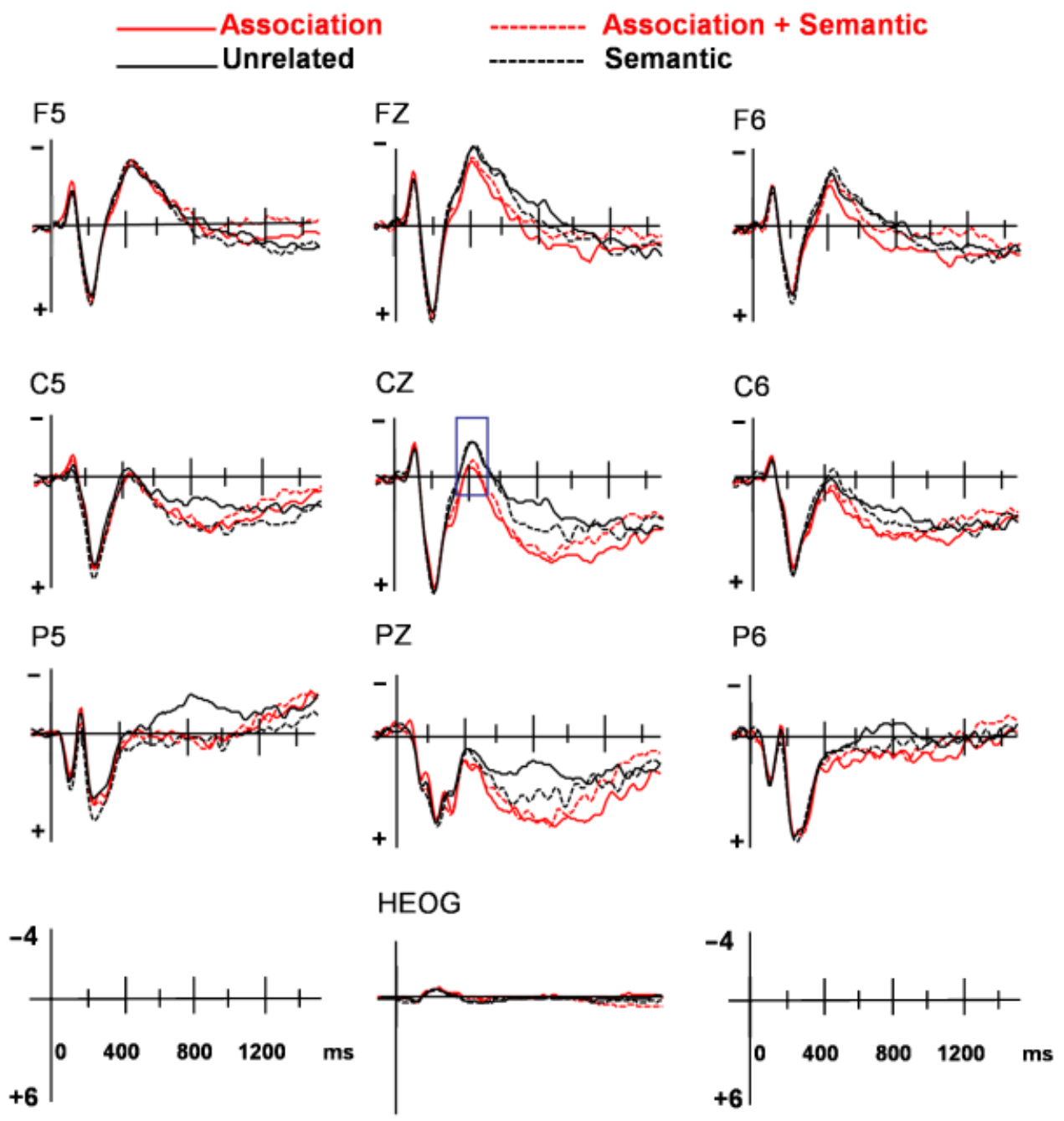

Figure 1. Grand average ERPs for association, association + semantic, semantic, and unrelated conditions shown from prestimulus to $1500 \mathrm{~ms}$ poststimulus. Ten electrodes are shown, nine electrode sites across frontal, central, and parietal scalp and the HEOG data. Negative voltage is plotted up. The box displayed on electrode $\mathrm{Cz}$ illustrates the time window typical of the N400 effects.

difference between the semantic and unrelated condition $(p>.05)$, as is clear from the overlap between the semantic and unrelated waveforms at central sites shown in Figures 1 and 2. A follow-up ANOVA also revealed a significant effect of Relationship Type at fronto-central medial sites, $F(3,72)=3.09$, $p<.03$, which pairwise comparisons showed reflected greater positive activity for the association and association + semantic conditions in contrast to the semantic condition (all $p \mathrm{~s}<.05$ ). Follow-up ANOVAs at medial, $F(2.1,50.1)=1.95, p>.05$, and lateral, $F(2,48.1)<1, p>.05$, centro-parietal sites failed to reach significance. The magnitude of the N400 effect is shown in Figure 6, illustrating both the maxima of the effect for the two association conditions at medial central sites and the absence of activity for the semantic condition. Collectively these analyses confirm reduced negativity for word pairs involving an associative relationship and not for those related purely by a semantic relationship (relative to a baseline of unrelated pairs).

500-900 ms. As Figure 1 shows, the reduced negativity for association and association + semantic conditions continues after $500 \mathrm{~ms}$, lasting until around $900 \mathrm{~ms}$. In addition, the waveforms for semantic and unrelated pairs also begin to diverge at around
$600 \mathrm{~ms}$. The activity seen for the semantic condition appears quite distinct from the earlier N400 effect, exhibiting a left parietal maxima. In fact, this left parietal effect can be seen for all three of the relationship types from around 500 to $1100 \mathrm{~ms}$, as shown in Figure 3 at electrode P5. As the primary focus of the current article concerns the N400 effect, the time window of 500$900 \mathrm{~ms}$ was chosen to capture the continuation of the N400 effect maximal across centro-parietal scalp (nonetheless, analysis using an extended 500-1100-ms time window revealed equivalent findings). The primary aim of the analyses during the later time window was to clarify whether there is (a) a continuation of the N400 effect for the associative and associative+ semantic conditions and (b) whether the N400 effect occurs for the semantic condition but is simply delayed.

An ANOVA was conducted with the factors of relationship type (association, association+semantic, semantic, unrelated), hemisphere (left, right) laterality (medial: sites 1 and 2; lateral: sites 5 and 6) and location (f, fc, c, cp, p, po lines) as shown in Figure 5. The ANOVA revealed a main effect of Relationship Type, $F(2.1,50.0)=7.6, p<.001$, and significant Relationship Type $\times$ Hemisphere, $F(3,72)=5.24, p<.003$, Relationship Type $\times$ Laterality, $F(2.4,57.8)=11.7, p<.001$, and Relationship 


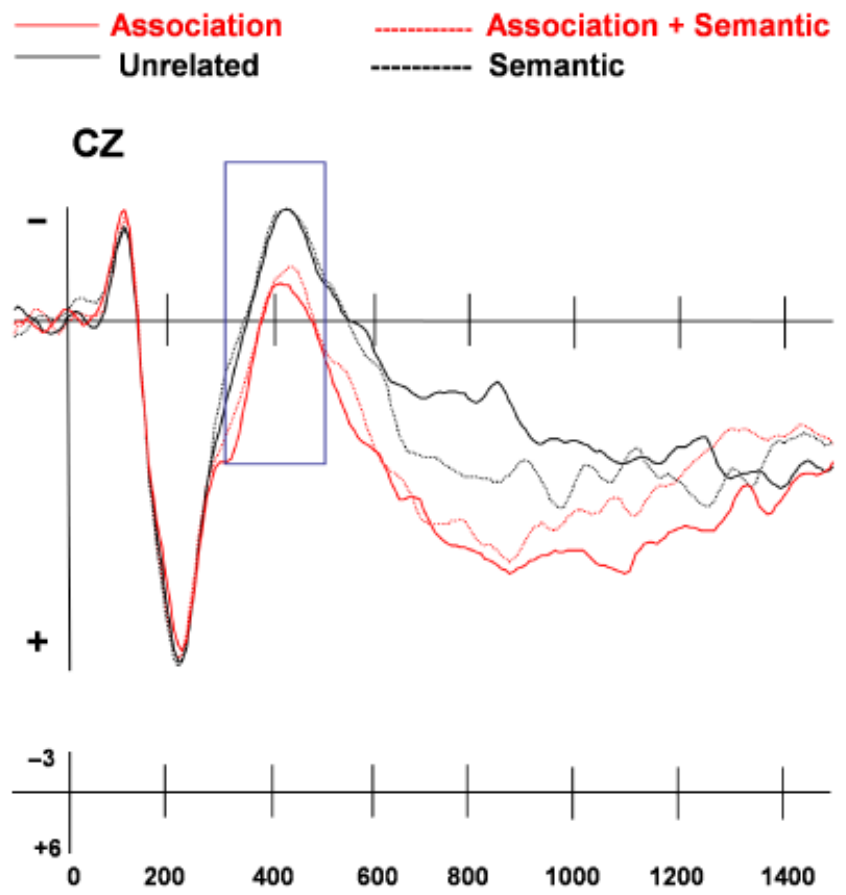

Figure 2. Grand average ERPs for association, association+semantic, semantic, and unrelated conditions at electrode $\mathrm{CZ}$, where effects are maximal. Data are shown as in Figure 1.

Type $\times$ Laterality $\times$ Location $, \quad F(7, \quad 168.7)=2.08, \quad p<.05$, interactions. The Relationship Type $\times$ Laterality $\times$ Location interaction was of primary interest and was followed up with subsidiary ANOVAs conducted separately on each of the six locations with factors of relationship type and laterality (collapsed across hemisphere). These analyses revealed that the original significant three-way interaction reflected Relatioship

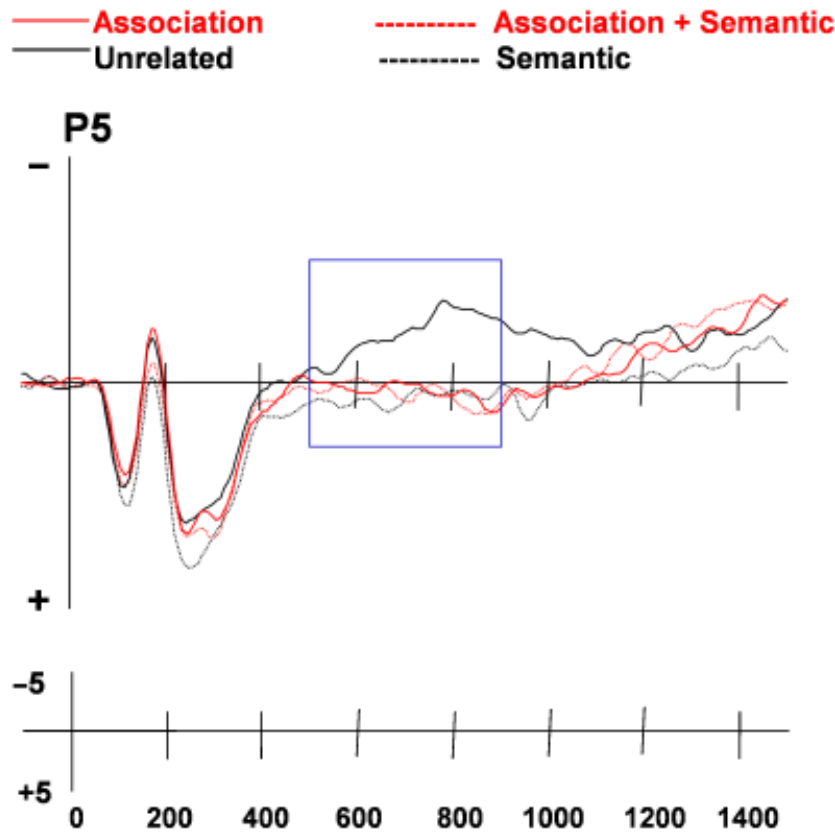

Figure 3. Grand average ERPs for association, association+semantic, semantic, and unrelated conditions at electrode P5. Data are shown as in Figure 1 . The box illustrates the $500-900-\mathrm{ms}$ time window.
Type $\times$ Laterality interactions at the frontal, $F(2.1,51.1)=3.43$, $p<.04$, fronto-central, $F(3,72)=13.12, p<.001$, central, $F(3,72)$ $=5.08, p<.003$, centro-parietal, $F(3,72)=9.23, p<.001$, parietal, $F(2.1,51.5)=5.64, p<.005$, and parietal-occipital, $F(3,72)$ $=4.42, p<.007$, locations. As can be seen from Figure 1 , these significant interactions across the scalp during this time window appear to reflect the presence of two effects: (a) continuation of the N400 effects observed for the association and association + semantic conditions at central medial electrodes during $300-500 \mathrm{~ms}$ and (b) the presence of a later onsetting ( $\sim 500 \mathrm{~ms})$ effect maximal at left posterior sites for all three related word pairs. Subsidiary ANOVAs were conducted separately on medial and lateral sites (collapsed across hemisphere) with the factor of relationship type (association, association+semantic, semantic, unrelated) at each of the six locations to substantiate this observation.

ANOVAs on medial sites revealed significant effects at the frontal, $F(3,72)=3.29, p<.03$, fronto-central, $F(3,72)=7.15$, $p<.001$, central, $F(2.2,52.9)=8.4, p<.001$, centro-parietal, $F(2.3,54.9)=9.6, p<.001$, parietal, $F(2.3,54.9)=8.35, p<.001$, and the parietal-occipital, $F(3,72)=7.79, p<.001$, locations. Pairwise comparisons revealed that significant effects at the frontal, fronto-central, central, and centro-parietal locations reflect greater positive activity for the association and association + semantic conditions in comparison to the unrelated condition (all $p \mathrm{~s}<.05$ ), with no significant differences between the semantic and unrelated conditions (all $p s>.05$ ). Thus, importantly, these analyses reveal continuation of the earlier N400 effects maximal at central medial sites observed during 300-500 $\mathrm{ms}$ for the association and association+semantic condition sites but, importantly, again reveal no significant effect for the semantic condition.

Main effects of Relationship Type for medial sites at the parietal and parietal-occipital locations reflect greater positive activity for all three related types in comparison to the unrelated condition (all $p s<.05$ ). This later onsetting effect is present for all three relationship types, is maximal at left posterior sites (see Figure 3), and clearly appears to reflect processing that is not specific to the presence of either association or a semantic relationship.

ANOVAs on lateral sites revealed significant effects at the central, $F(3,72)=3.65, p<.03$, centro-parietal, $F(2.3,54.3)=$ $4.1, p<.02$, parietal, $F(3,72)=7.6, p<.001$, and the parietaloccipital, $F(3,72)=7.19, p<.001$, locations. Pairwise comparisons revealed that the significant effect of Relationship Type at both central and centro-parietal lateral sites reflects greater positive activity for the association and association + semantic conditions in comparison to the unrelated condition (all $p s<.05$ ), with no significant difference between the semantic and unrelated conditions (all $p s>.05$ ). In contrast, pairwise comparisons revealed that the significant effect of Relationship Type at both parietal and parietal-occipital lateralized sites reflects greater positive activity for all three related types in comparison to the unrelated condition (all $p \mathrm{~s}<.05$ ), reflecting an effect that has both a later onset than the N400 effect and different maxima. This effect is present for all three relationship types after $500 \mathrm{~ms}$ and is maximal at left posterior sites (see Figure 3). The topographic distribution of effects is shown in Figure 4.

\section{Behavioral Test Data}

Accuracy data from the recognition test were analyzed using an ANOVA with a factor of relationship type (association, 


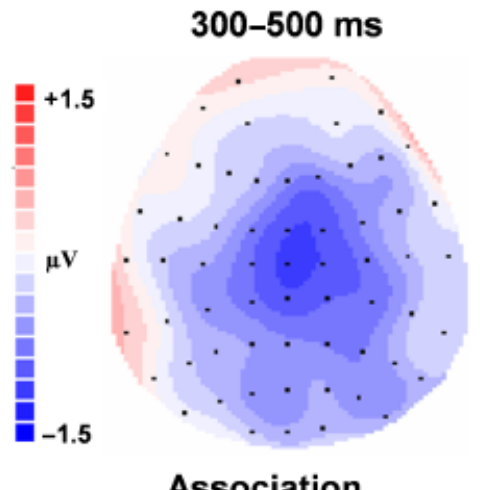

Association

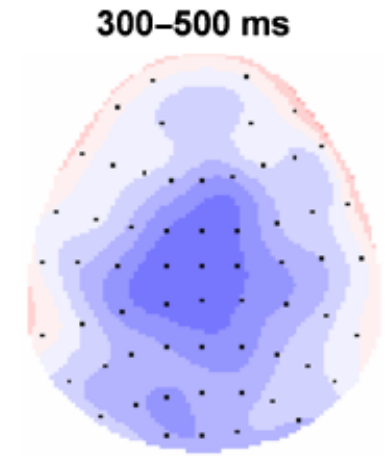

Association + Semantic

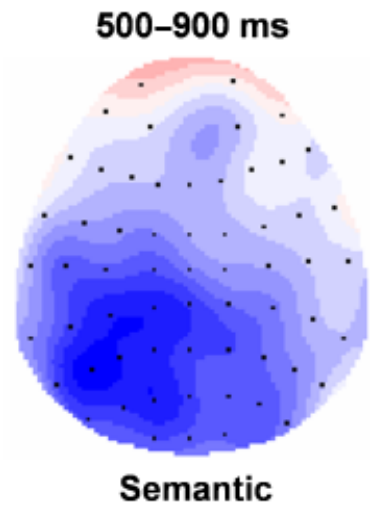

Semantic

Figure 4. Topographic maps showing the distribution of the N400 effect (each related condition subtracted from the unrelated condition) for association and association + semantic conditions during the 300-500-ms latency window and for the semantic condition during the 500-900-ms latency window. Although equivalent N400 effects are present for the two associative conditions during the early time window, a distinct left parietal pattern of activity is seen for the semantic condition.

association+semantic, semantic, unrelated). This analysis revealed a significant effect of Relationship Type, $F(3,72)=43.4$, $p<.001$. Pairwise comparisons revealed graded performance; each relationship type was significantly different from all others $(p<.05$ in each case), with greatest recognition for associative pairs and poorest for unrelated pairs (see Table 3 ). Reaction time data for correct responses were analyzed using an ANOVA with a factor of relationship (association, association + semantic, semantic, unrelated), revealing a significant effect of Relationship Type, $F(3,72)=46.28, p<.001$. Pairwise comparisons revealed graded performance, with slower reaction times for the unrelated condition than all other conditions $(p<.001)$ and slower reaction times for the semantic condition than the associative $(p<.001)$ and associative + semantic $(p<.02)$ conditions. There was no significant difference in reaction times between the associative and the associative + semantic conditions $(p>.05)$.

\section{Discussion}

The present study independently manipulated the presence of associative and semantic relationships while examining the N400 effects elicited during a conscious encoding task. The findings suggest that associative and semantic relationships are neurally dissociable. Specifically, modulation of the N400 was observed for word pairs related by association (with and without an addi-

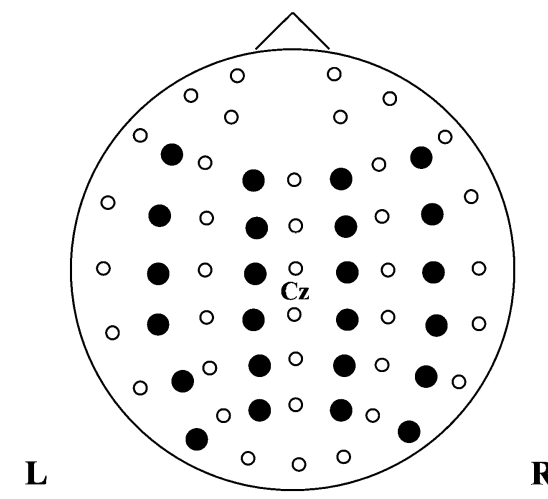

Figure 5. Illustration of the electrodes used to characterize the N400 effect during $300-500 \mathrm{~ms}$ as well as both the continuation of the N400 effect during 500-900 $\mathrm{ms}$ and the onset of the left posterior effect. tional semantic relationship) whereas no modulation of the N400 was observed for pairs related solely by a semantic relationship. Analyses revealed an effect that was maximal over superior central electrodes for association and association + semantic word pairs relative to unrelated pairs, consistent with the traditional N400 effect that was absent for the semantic condition. Given the widely accepted view that the N400 effect provides an index of the degree to which information is processed for meaning, these findings suggest that it is the associations between words, rather than the semantic relationship between them, that is critical for the processing of meaning, at least for the task demands employed in the current study.

The difference between a semantic relationship with and without association appears slight, but we show that this subtlety (that lemon calls to mind orange but that cereal does not call to mind bread by free association) is crucial in the processing of meaning during an explicit language comprehension task. The current findings do not, of course, argue against the importance of semantic information per se. As noted in the introduction, for example, data from neuropsychological patient studies provide

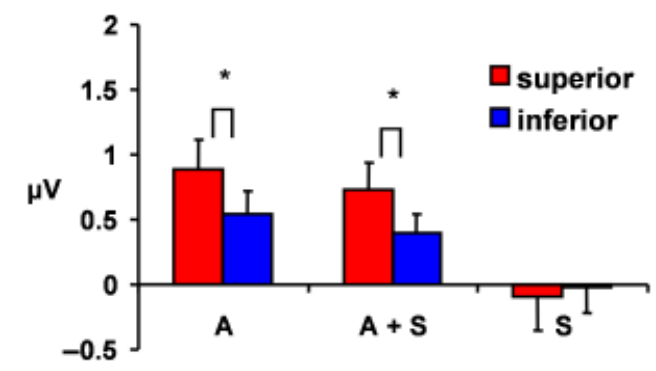

Figure 6. The N400 effects were maximal at superior central electrodes. The histogram shows the magnitude of effects for association, association+semantic, and semantic conditions, respectively (each subtracted from the unrelated condition) collapsed across a set of superior (red bars, online version; light gray bars, print version) and inferior (blue bars, online version; dark gray bars, print version) groupings. Superior electrodes include FC1, FCZ, FC2, C2, CP2, CPZ, CP1, and $\mathrm{C} 1$ and inferior electrodes include F3, FZ, F4, C4, P4, PZ, P3, C3. Error bars shown represent standard error of the mean. This figure illustrates (a) the presence of an N400 effect for the association conditions that is larger over superior sites, reflecting the characteristic superior central distribution of the N400 effect, and (b) the absence of an N400 effect for the semantic condition. 
Table 3. Memory Retrieval Data

\begin{tabular}{lcc}
\hline \hline Relationship type & Memory accuracy & Reaction time \\
\hline Association & $80.8(11.6)$ & $1241.2(130)$ \\
Association+semantic & $78.3(11.5)$ & $1259.5(134)$ \\
Semantic & $74.4(13.3)$ & $1293.9(133)$ \\
Unrelated & $61.6(15.4)$ & $1379.5(139)$ \\
\hline \hline
\end{tabular}

Data show the mean percentage of word pairs that were correctly identified as being old, and reaction times for these responses, during a subsequent recognition memory test. Standard deviations are shown in parentheses.

compelling evidence for specific semantic knowledge stores within the brain, whereby information is organized categorically (e.g., see Capitani et al., 2003). Nonetheless, our findings demonstrate that when presented with information that shares a semantic relationship and is associated, meaningful information is first extracted from the associations between items.

The findings presented here build on, but also challenge, current functional interpretations of the N400 effect (e.g., Bentin et al., 1995; Federmeier \& Kutas, 1999; Heinze et al., 1998; Kuonios \& Holcomb, 1992; Kutas, 1993; Kutas \& Hillyard, 1980, 1984; Kutas \& Iragui, 1998; Kutas et al., 1984; Luck et al., 1996; Neville et al., 1986) by demonstrating the specific type of relationship that is necessary to elicit the effect, at least during a task that encourages conscious processing. Although we report these findings from a single task that did not promote expectancy, there is evidence to suggest that this finding will extend to other tasks. First, studies that have compared the N400 effects elicited during the presentation of related words (typically related by both associative and semantic relationships) at varying SOAs have revealed N400 effects for words presented simultaneously that is similar to those for those following a delay (e.g., Anderson \& Holcomb, 1995). Second, Koivisto and Revonsuo's (2001) findings suggest that a semantic relationship is not sufficient to elicit the N400 effect during a priming task. We extend the findings of the Koivisto and Revonsuo study to an explicit language comprehension task paradigm that encouraged conscious processing. In addition, the inclusion of our combined associative + semantic condition (absent in the Koivisto and Revonsuo study) enabled us to show that this condition elicited N400 effects equivalent to those for association alone, suggesting that it is associative relationships that are critical in eliciting the N400 effects reported within the literature. This latter finding is noteworthy because the associative + semantic stimuli are most similar to the stimuli typically used in N400 language comprehension studies. Of course, our study employed word pairs as stimuli, and further research is required to investigate whether equivalent results would be found within experiments using sentences as contexts for the processing of meaningful information.

By controlling for the contribution of association we show that meaning derived from a semantic relationship is not sufficient to produce the N400 effect, at least under conditions of conscious processing. Our findings suggest the possibility that the N400 effect reflects the processing of meaning that results from the associations between words. A number of studies examining the N400 effect have manipulated the degree of typicality of category exemplar strength (Heinze et al., 1998; Nunez-Pena \& Honrubia-Serrano, 2005). These studies report smaller (Heinze et al., 1998) or no (Nunez-Pena \& Honrubia-Serrano, 2005) re- ductions in negativity when exemplars were less typical. Thus, the absence of a reduction in negativity reported here for word pairs characterized by a semantic relationship suggests the possibility that small or absent reductions in negativity reported in the literature for less typical members (Heinze et al., 1998; Nunez-Pena \& Honrubia-Serrano, 2005) may reflect the unmeasured confound of differences in the strength of association between the words used to represent typical and atypical category members. Further research, designed to specifically investigate categorical semantic memory while independently manipulating association and typicality of exemplars, is warranted to directly test this hypothesis.

Our data also contribute to neuropsychological evidence that suggests a neural dissociation between processing of semantic and associative representations. We support the dissociation reported for activations of semantic and associative representations in patients with Alzheimer's disease (Glosser \& Friedman, 1991) and children with poor reading comprehension (Nation \& Snowling, 1999). Debate is ongoing within the neuropsychological literature as to whether dissociation between different types of relationships in semantic memory reflects "disrupted access" to the specific relationship type or "storage deficits" of a representational nature. For example, dissociation between functional and categorical properties in a patient with semantic dementia (Moss, Tyler, Hodges, \& Patterson, 1995) has been interpreted as reflecting impaired access to functionally encoded concepts while the representations of functional and categorical information remain intact. Dissociation of semantic and associative information in patients with Alzheimer's disease, in contrast, has been interpreted as suggesting the existence of at least two networks of relationships in the lexicon, whereby representations are differentially disrupted (Glosser \& Friedman, 1991). This interpretation of two networks is supported by popular theory of lexical processing (Collins \& Loftus, 1975); activation of associations is believed to occur because connections made through repeated occurrence of two words lead to representations at the lexical level. The present findings in a nonpatient sample where representations are presumably intact suggests, however, that independent routes of access exist within the brain.

An alternative account of the current findings is that the N400 effect seen for the associative only condition reflects encoding of the words as a unitized representation. Indeed, it is reasonable to assume that associative word pairs (like traffic-jam) could be perceived as a single unit. Clearly, comparison of the N400 effect elicited for single and compound words is an important priority for future research. However, a unitization account is unlikely to account for our findings of an N400 modulation for the associative + semantic word pairs. Words in the associative + semantic condition are unlikely to be associated with a unitized representation (e.g., lemon-orange), but the N400 effects for the associative and associative + semantic conditions were equivalent. Perception of the word pairs as a unit, therefore, does not adequately account for the pattern of N400 effects reported here.

Finally, we turn from the N400 effect to briefly consider the later onsetting left parietal activity. During the 500-900-ms time window a left lateralized posterior effect was observed for all three relationship types (including the semantic only condition) relative to the unrelated condition. The fact that the later parietal effect is present for all three relationship types suggests that it is not specific to the processing of semantic or associative information. The effect may simply reflect the specific task employed in the present study; perhaps subjects first detected any associa- 
tive relationship between the words and, following this, looked for any other cues that could facilitate memory encoding.

In fact, this late posterior effect may be better characterized as a relative negativity for the unrelated word pairs compared to all other conditions, suggesting a process that is sensitive to the presence of unrelated word pairs. One possible interpretation of the later posterior effect is that it reflects retrieval of previous experience of the related words. To all appearances the timing and topography of this later effect is similar to that of the leftparietal effect old/new effect associated with the engagement of recollection, an episodic retrieval process (cf. Donaldson \& Rugg, 1998, 1999; Greve, van Rossum, \& Donaldson, 2007; Rhodes \& Donaldson, 2007; Wilding \& Rugg, 1996). It is, of course, impossible for us to confirm that the same neural generators are responsible for the left parietal old/new effect and the late posterior effect seen here, making any conclusions tentative at present. Nonetheless, the presence of this effect for all three relationship types (relative to the unrelated word pairs) points toward the intriguing possibility that it may reflect recollection of previous experiences of the related pairs from long-term memory (a process that would be less likely to occur for unrelated pairs).

Our findings highlight the utility of ERP measures for studying the comprehension of language and, indeed, cognitive processes in general. We were able to show dissociation between associative and semantic relationships because of the ability of ERPs to provide a real-time record of neural activity. The present study shows the value of using this method to identify and dissociate cognitive processes on the basis of fine-grained temporal information. Further research is warranted to investigate whether the specific task conditions in the present study are responsible for the finding of dissociation between associative and semantic representations.

\section{REFERENCES}

Anaki, D., \& Henik, A. (2003). Is there a "strength effect" in automatic semantic priming? Memory and Cognition, 31, 262-272.

Anderson, J. E., \& Holcomb, P. J. (1995). Auditory and visual semantic priming using different stimulus onset asynchronies: An event-related brain potential study. Psychophysiology, 32, 177-190.

Bentin, S., Kutas, M., \& Hillyard, S. (1995). Semantic processing and memory for attended and unattended words in dichotic listening: Behavioural and electrophysiological evidence. Journal of Experimental Psychology: Human Performance, 21, 54-67.

Capitani, E., Laiacona, M., Mahon, B., \& Caramazza, A. (2003). What are the facts of semantic category-specific deficits? A critical review of the clinical evidence. Cognitive Neuropsychology, 20, 213-261.

Collins, A. M., \& Loftus, E. F. (1975). A spreading activation theory of semantic processing. Psychological Review, 82, 407-428.

Coulson, S., Federmeier, K. D., Van Petten, C., \& Kutas, M. (2005). Right hemisphere sensitivity to word and sentence level context: Evidence from event related brain potentials. Journal of Experimental Psychology: Learning Memory and Cognition, 31, 129-147.

Donaldson, D. I., \& Rugg, M. D. (1998). Recognition memory for new associations: Electrophysiological evidence for the role of recollection. Neuropsychologia, 36, 377-395.

Donaldson, D. I., \& Rugg, M. D. (1999). Event-related potential studies of associative recognition and recall: Electrophysiological evidence for context dependent retrieval processes. Cognitive Brain Research, 8 , $1-16$.

Federmeier, K. D., \& Kutas, M. (1999). A rose by any other name: Longterm memory structure and sentence processing. Journal of Memory and Language, 41, 469-495.

Ferrand, L., \& New, B. (2003). Semantic and associative priming in the mental lexicon. In P. Bonin (Ed.), Mental lexicon: Some words to talk about words (pp. 25-43). Hauppauge, NY: Nova Science Publisher.

Glosser, G., \& Friedman, E. B. (1991). Lexical but not semantic priming in Alzheimer's disease. Psychology and Aging, 6, 522-527.

Greve, A., van Rossum, M., \& Donaldson, D. I. (2007). Investigating the functional interaction between semantic and episodic memory: Convergent behavioural and electrophysiological evidence for the role of familiarity. NeuroImage, 34, 801-814

Heinze, H., Muente, T., \& Kutas, M. (1998). Context effects in a category verification task as assessed by event-related brain potential (ERP) measures. Biological Psychology, 47, 121-135.

Hodgson, J. M. (1991). Informational constraints on pre-lexical priming. Language and Cognitive Processes, 6, 169-205.

Huettig, F., Quinlan, P. T., McDonald, S. A., \& Altmann, G. T. M. (2006). Models of high-dimensional semantic space predict languagemediated eye movements in the visual world. Acta Psychologica, 121, 65-80.

Jasper, H. H. (1958). Report to the committee on methods of clinical examination in electroencephalography. Appendix: the ten-twenty system of the International Federation. Electroencephalography and Clinical Neurophysiology, 10, 370-375.
Kiefer, M. (2002). The N400 is modulated by unconsciously perceived masked words: Further evidence for an automatic spreading activation account of N400 priming effects. Cognitive Brain Research, 13, 27-39.

Koivisto, M., \& Revonsuo, A. (2001). Cognitive representations underling the N400 priming effect. Cognitive Brain Research, 12, 467-490.

Kucera, H., \& Francis, W. N. (1967). Computational analysis of presentday American English. Providence, RI: Brown University Press.

Kuonios, J., \& Holcomb, P. J. (1992). Structure and process in semantic memory: Evidence from event-related brain potentials and reaction times. Journal of Experimental Psychology: General, 121, 459-479.

Kutas, M. (1993). In the company of other words: Electrophysiological evidence for single-word and sentence context effects. Language and Cognitive Processes, 8, 533-572.

Kutas, M., \& Federmeier, K. D. (2000). Electrophysiology reveals semantic memory use in language comprehension. Trends in Cognitive Neuroscience, 412, 463-470.

Kutas, M., \& Hillyard, S. A. (1980). Reading senseless sentences: Brain potentials reflect semantic incongruity. Science, 207, 203-205.

Kutas, M., \& Hillyard, S. A. (1984). Brain potentials during reading reflect word expectancy and semantic association. Nature, 307, $161-163$.

Kutas, M., \& Iragui, V. (1998). The N400 in a semantic categorization task across 6 decades. Electroencephalography and Clinical Neurophysiology, 108, 456-471.

Kutas, M., Lindamood, T. E., \& Hillyard, S. A. (1984). Word expectancy and event-related potentials during sentence processing. In S. Kornblum \& J. Requin (Eds.), Preparatory states and processes (pp. $217-$ 237). Hillsdale, NJ: Erlbaum.

Luck, S. J., Vogel, E. K., \& Shapiro, K. L. (1996). Word meanings can be accessed but not reported during the attentional blink. Nature, 383, 616-618.

McDonald, S. (2006). Semantic space model demo. Retrieved from http:// www.iccs.inf.ed.ac.uk/ smcdonal/semantic_space_model.html.

McRae, K., \& Boisvert, S. (1998). Automatic semantic similarity priming. Journal of Experimental Psychology: Learning, Memory and Cognition, 24, 558-572.

Moss, H. E., Ostrin, R. K., Tyler, L. K., \& Marslen-Wilson, W. D. (1995). Accessing different types of lexical semantic information: Evidence from priming. Journal of Experimental Psychology: Learning Memory and Cognition, 21, 863-883.

Moss, H. E., Tyler, L. K., Hodges, J. R., \& Patterson, K. (1995). Exploring the loss of semantic memory in semantic dementia: Evidence from a primed monitoring study. Neuropsychology, 9, $16-26$.

Nation, K., \& Snowling, M. J. (1999). Developmental differences in sensitivity to semantic relations among good and poor comprehenders: Evidence from semantic priming. Cognition, 70, B1-B13.

Neville, H. J., Kutas, M., Chesney, G., \& Schmidt, A. (1986). Eventrelated brain potentials during initial encoding and subsequent 
recognition memory of congruous and incongruous words. Journal of Memory and Language, 25, 75-92.

Nunez-Pena, I. M., \& Honrubia-Serrano, L. M. (2005). N400 and category exemplar associative strength. International Journal of Psychophysiology, 56, 45-54.

Perea, M., Gotor, A., \& Nacher, M. J. (1997). Efectos de facilitation associativa vs. semantica con una breve asincronia estimular senaltest. [Associative and semantic priming effects at a very brief stimulus onset asynchrony]. Psicothema, 9, 509-517.

Perea, M., \& Rosa, E. (2002). The effects of associative and semantic priming in the lexical decision task. Psychological Research, 66, 180-194.

Postman, L., \& Keppel, G. (1970). Norms of word association. New York: Academic Press.

Rhodes, S. M., \& Donaldson, D. I. (2007). Electrophysiological evidence for the influence of unitization on the processes engaged during episodic retrieval: Enhancing familiarity based remembering. Neuropsychologia, 45, 412-424.

Scan 4.2, Volume II. (2001). Sterling, VA: Neurosoft Inc.
Semlitsch, H. V., Anderer, P., Schuster, P., \& Presslich, O. (1986). A solution for reliable and valid reduction of ocular artefacts applied to the P300 ERP. Psychophysiology, 23, 695-703.

Shelton, J. R., \& Martin, R. C. (1992). How semantic is automatic semantic priming? Journal of Experimental Psychology: Learning, Memory and Cognition, 18, 1191-1210.

Thompson-Schill, S. L., Kurtz, K. J., \& Gabrieli, D. (1998). Effects of semantic and associative relatedness on automatic priming. Journal of Memory and Language, 38, 440-458.

Word Association Thesaurus (n.d.). Edinburgh Association Thesarus, MRC Psycholinguistic database. Retrieved January 5, 2005, from http://www.eat.rl.ac.uk/

Wilding, E. L., \& Rugg, M. D. (1996). An event-related potential study of recognition memory with and without retrieval of source. Brain, $119,889-905$.

Williams, J. N. (1996). Is automatic priming semantic? European Journal of Cognitive Psychology, 22, 139-151.

(Received September 8, 2006; ACCEPTEd June 22, 2007) 